\title{
Biomarker assays: is it time for a standalone regulatory guidance?
}

\author{
Steve Keller ${ }^{*, 1}$ \& Shobha Purushothama ${ }^{2}$ \\ ${ }^{1}$ AbbVie Biotherapeutics 1500 Seaport Blvd Redwood City, CA 94063, USA \\ ${ }^{2}$ Biogen, 225 Binney Street, Cambridge, MA 02142, USA \\ *Author for correspondence: Steve.Keller@abbvie.com
}

\section{"Biomarkers have come of age and all would agree that the methods we use to measure them 'are not PK assays'. "”}

First draft submitted: 19 October 2018; Accepted for publication: 26 October 2018; Published online: 16 November 2018

Keywords: biomarker assay $\bullet$ context of use $\bullet$ fit for purpose $\bullet$ relative accuracy $\bullet$ parallelism $\bullet$ surrogate matrix

One cannot attend a contemporary conference in which biomarker methods and data are discussed without hearing, 'biomarker assays are not PK assays' at least a few times. And neither of these authors has ever heard the response, 'yes they are'. In fact, if you have worked on biomarker assays and spent time thinking about such things, you are likely to nod and start listing the reasons for such a statement, at least mentally. So, they are not the same and serve different purposes, yet share similarities. But just how closely related are these classes of bioanalytical assays? This question warrants careful consideration because the implications of that relationship have important consequences.

Stating the obvious, PK assays serve to measure drug concentration in samples to establish the PK properties of a novel xenobiotic in circulation or at the site(s) of interest. The utility of biomarker measurements can include characterizing the pharmacodynamic (PD) properties of drugs (e.g., pathway activation/suppression), confirming mechanism of action (target engagement), providing insights into toxicity, differentiation from competitor compounds and, in some cases, biomarkers can be essential as companion diagnostics for the safe and effective use of lifesaving therapies. One might argue that PK assays are more limited in purpose, but it is important to acknowledge that PK data are always critically important for the registration of novel therapies, while biomarker data rarely hold such significance. In fact, the vast majority of biomarker data is used for internal decision making purposes, and never finds its way into regulatory filings. The purposes for these assays are only connected by the need to understand the pharmacology of drugs.

\section{PK versus biomarker assays: similarities $\&$ differences}

What about the methods themselves? It is certainly true that one is likely to encounter many challenges in developing and validating ligand-binding PK and biomarker methods, and some of these challenges are shared. For example, each require working in biological (and at times troublesome) matrices, achieving adequate sensitivity/specificity, and securing adequate supplies of key reagents, to name only a few common hurdles. These classes of bioanalytical methods also share technology platforms, similarity of basic protocols, and even training. We all know talented bioanalytical scientists that work on PK assays for part of their career (or the month), then make the switch to biomarker assays and back again (while tackling an occasional immunogenicity assay).

But how are these methods different? PK assay work includes some additional complexities, such as how to handle emerging novel drug modalities (e.g., gene therapies, multitargeted agents, fusion molecules, antibody-drug conjugates and novel probodies) and the effects of metabolism on the analyte. Biomarker methods are complicated by the use of commercial kits versus homegrown proprietary methods, understanding specificity of reagents and the presence of endogenous analyte in matrices, resulting in the need to use surrogate matrices. A key difference is the fact that very few true standards exist for biomarker methods, making 'relative accuracy' the norm; a concept that is not yet well understood. Biomarkers can be measured in multiplex formats to allow simultaneous quantitation of multiple analytes from a single, precious sample. Although it is true that multiplex PK methods are becoming 
more common in our industry, biomarker multiplexing is probably more common and certainly more extensive with respect to the number of analytes simultaneously measured. This introduces complexities around establishing multiple quantitative ranges, and how to handle run failure of a subset of analytes (i.e., establishing practices for retesting that result in multiple acceptable values for some analytes). Due to the lack of true standards exist for biomarker methods, establishing parallelism becomes key to understanding biomarker assay performance and defining the context of use. In addition, accuracy/precision acceptance criteria may adjust based on anticipated effect size changes. This list of difference is admittedly incomplete, but captures some of the more important distinctions.

So, what do these comparisons tell us about the relationship between these methods? While similarities exist, the many differences of application and methodology make PK and biomarker methods seem more like distant cousins than identical twins.

\section{Current state of affairs}

It is not surprising that over the past dozen years many useful biomarker white papers have been published [1-5]. These publications have played an important role in creating a conceptual framework and language for biomarker method discussions (e.g., fit-for-purpose and context of use); and in establishing (or at least recommending) best practices for developing, validating and conducting biomarker sample testing using commercial kits [6], custom ligand-binding assays [7] and flow cytometry [8-10], multiplexing [11]. Although these white papers at least partially fill a void and represent an important step forward, there is still lingering confusion around best practices and what, exactly, constitutes an acceptable approach for validating biomarker methods used for exploratory or advanced drug development purposes.

A glimpse into the regulatory thought process around biomarkers came this year when the US FDA issued an updated Bioanalytical Method Validation guidance a nod to the similarities we allude to, while acknowledging differences [12]. Biomarkers are mentioned in the main body of the document, although there is clearly a more significant focus on PK method development and validation. Importantly, biomarkers get a couple pages of dedicated attention in the 'additional issues' section. However, relegation to a section with that title, is inadequate to address the inherent complexities posed by biomarker assays and raises the question: is it time for a dedicated biomarker bioanalytical method validation guidance document?

Biomarkers have come of age and all would agree that the methods we use to measure them 'are not PK assays'. Some participants at the 2018 WRIB subscribe to the school of thought that a standalone biomarker guidance document would be extremely useful. In fact, our immunogenicity colleagues (antidrug antibodies are in response to therapy [13]) benefit greatly from regulatory agency issued dedicated, comprehensive guidance documents from around the globe. One could argue that a dedicated biomarker regulatory guidance is impractical due to the breadth of use of biomarker data. There does seem to be a consensus that having more clarity in terminology such as context of use with illustrative case study examples in White Papers or Conference Proceedings will be beneficial to the bioanalytical community. Whether one believes that a dedicated biomarker guidance document would be warranted or not, there is clearly a need for discussion and knowledge sharing in the interest of clarifying best practices for biomarker methods across our industry. Discussions and debate to reach consensus take time, so when can we start?

\section{Financial \& competing interests disclosure}

The authors have no relevant affiliations or financial involvement with any organization or entity with a financial interest in or financial conflict with the subject matter or materials discussed in the manuscript. This includes employment, consultancies, honoraria, stock ownership or options, expert testimony, grants or patents received or pending, or royalties.

No writing assistance was utilized in the production of this manuscript.

\section{References}

1. Gupta S, Richards S, Amaravadi L et al. 2017 White Paper on recent issues in bioanalysis: a global perspective on immunogenicity guidelines \& biomarker assay performance (Part 3 - LBA: immunogenicity, biomarkers and PK assays). Bioanalysis 9(24), 1967-1996 (2017).

2. Richards S, Amaravadi L, Pillutla R et al. 2016 White Paper on recent issues in bioanalysis: focus on biomarker assay validation (BAV): (Part 3 - LBA, biomarkers and immunogenicity). Bioanalysis 8(23), 2475-2496 (2016). 
3. Lee JW, Devanarayan V, Barrett YC et al. Fit-for-purpose method development and validation for successful biomarker measurement. Pharm. Res. 23(2), 312-328 (2006).

4. Lee JW. Method validation and application of protein biomarkers: basic similarities and differences from biotherapeutics. Bioanalysis 1(8), 1461-1474 (2009).

5. Amur S, Frueh FW, Lesko LJ, Huang SM. Integration and use of biomarkers in drug development, regulation and clinical practice: a US regulatory perspective. Biomark. Med. 2(3), 305-311 (2008).

6. Khan MU, Bowsher RR, Cameron $\mathrm{M}$ et al. Recommendations for adaptation and validation of commercial kits for biomarker quantification in drug development. Bioanalysis 7(2), 229-242 (2015).

7. Cowan K, Gao X, Parab V et al. Fit-for-purpose biomarker immunoassay qualification and validation: three case studies. Bioanalysis 8(22), 2329-2340 (2016).

8. O'Hara DM, Xu Y, Liang Z, Reddy MP, Wu DY, Litwin V. Recommendations for the validation of flow cytometric testing during drug development: II assays. J. Immunol. Methods 363(2), 120-134 (2011).

9. Green CL, Stewart JJ, Hogerkorp CM et al. Recommendations for the development and validation of flow cytometry-based receptor occupancy assays. Cytometry B Clin. Cytom. 90(2), 141-149 (2016).

10. Sommer U, Morales J, Groenewegen A et al. Implementation of highly sophisticated flow cytometry assays in multicenter clinical studies: considerations and guidance. Bioanalysis 7(10), 1299-1311 (2015).

11. Jani D, Allinson J, Berisha F et al. Recommendations for use and fit-for-purpose validation of biomarker multiplex ligand binding assays in drug development. AAPS J. 18(1), 1-14 (2016).

12. US FDA, CDER, CVM, Bioanalytical method validation: guidance for industry (2018). http://www.fda.gov/downloads/drugs/guidances/ucm070107

13. Allinson JL. Clinical biomarker validation. Bioanalysis 10(12), 957-968 (2018). 
